\title{
Excited Heavy Baryons and Their Symmetries II: Effective Theory
}

\author{
Chi-Keung Chow, Thomas D. Cohen and Boris A. Gelman \\ Department of Physics, University of Maryland, College Park, MD 20742-4111.
}

\begin{abstract}
We develop an effective theory for heavy baryons and their excited states. The approach is based on the contracted $O(8)$ symmetry recently shown to emerge from QCD for these states in the combined large $N_{c}$ and heavy quark limits. The effective theory is based on perturbations about this limit; a power counting scheme is developed in which the small parameter is $\lambda^{1 / 2}$ where $\lambda \sim$ $1 / N_{c}, \Lambda / m_{Q}$ (with $\Lambda$ being a typical strong interaction scale). We derive the effective Hamiltonian for strong interactions at next-to-leading order. The next-to-leading order effective Hamiltonian depends on only two parameters beyond the known masses of the nucleon and heavy meson. We also show that the effective operators for certain electroweak transitions can be obtained with no unknown parameters at next-to-leading order.
\end{abstract}

\section{INTRODUCTION}

Recently, it was pointed out that QCD when restricted to the space of heavy baryons (i.e. states with baryon number one containing a single heavy — b or c - quark) has a contracted $U(4)$ [1] or, more generally, $O(8)$ symmetry which emerges in the combined large $N_{c}$ (the number of colors) and heavy quark limits [2]. Of course, in the real world neither $N_{c}$ nor $m_{Q}$ (the generic heavy quark mass) is infinite. Nevertheless if they are large enough, QCD will possess an approximate symmetry. Approximate symmetries can provide significant phenomenological information about a system and have had a long and distinguished history in strong interaction physics. Effective field theory (EFT) is a powerful tool that can be used to extract this information. In this article we develop an effective theory to describe heavy baryons based on the approximate contracted $O(8)$ symmetry. This is important since this allows one to make testable predictions about the phenomenology of heavy baryons. This paper serves the role of bridging the formal developments of Ref. [2] with phenomenological descriptions of heavy baryons. Our ultimate goal is to study the spectroscopy and electroweak decays of heavy baryon states which will be done in Ref. [3].

Before discussing how to implement the EFT approach in the context of heavy baryons it is useful to recall how the EFT program works. Effective field theory is useful when there is a separation of scales between the low energy phenomena and higher energy physics. The short-distance physics can greatly influence the long-distance physics. However, when working at low momentum one cannot resolve the details of what is happening at short distances. The idea of EFT is to describe the low-momentum phenomena in a manner which is insensitive to the details of short-distance physics. Formally, one would begin with the full theory and integrate out all of the short-distance degrees of freedom in a functional integral. This yields an effective action to be used in a functional integral over the low momentum (long-distance) degrees of freedom. Clearly, the effective action so obtained cannot be expressed as the integral over a local Lagrangian. However, all of the nonlocalities occur at the scale of the short-distance degrees of freedom which have been integrated. Thus, to good approximation the effective action can be written in terms of a local Lagrangian. The effects of the short-distance physics are now contained in the coefficients in the effective Lagrangian.

Clearly such an approach is, at best, approximate. Fortunately, if the scale separation between the long-distance scales and the short-distance scales is large the approximation can be very good. Formally, one can develop a systematic power-counting expansion where the parameter is the ratio of the typical long-distance scale to the typical short-distance scale. As a practical matter one must truncate the effective Lagrangian at some order. The power counting, however, ensures that the effects of the neglected terms are formally down from the leading term by some power in the ratio of the scales. This, in turn, ensures that the principal effects of the short-distance physics are contained in a few coefficients in the effective Lagrangian. In this manner an insensitivity to the details of the shortdistance physics is achieved. The low momentum phenomenology depends on a few coefficients summarizing the effects of the short-distance physics; the manner by which the short-distance physics produces these coefficients is irrelevant.

In principle, all of the coefficients in the effective Lagrangian can be calculated from the underlying physics. Often, however, the underlying physics is either unknown or is calculationally intractable. EFT, nevertheless provides a conceptual framework for making predictions about the system. The key insight is that one can treat the parameters in the effective Lagrangian as phenomenological inputs fit from experiment. Thus, providing there are more pieces of 
independent data than there are free parameters at a given order, one can make real, albeit approximate predictions. Chiral perturbation theory is a familiar paradigm for the EFT program.

Let us now turn to the problem of heavy baryons. For simplicity we restrict our attention here to the problem of isoscalar heavy baryons - the $\Lambda_{b}$ and $\Lambda_{c}$ (generically $\Lambda_{Q}$ ) and their excited states. The generalization to include non-isoscalars such as the $\Sigma_{b}$ or $\Sigma_{c}$ raises no new conceptual issues but complicates the analysis. The approach of Ref. [2] begins with a restriction to a heavy baryon subspace of the full QCD Hilbert space, i.e. we consider only the space of states with baryon number one and one heavy quark. The physical picture that emerges from the modeldependent analysis of Ref. [1] 2] is that low-lying states of the heavy baryon in the combined large $N_{c}$ and heavy quark limits can be represented as harmonic collective motion of the "brown muck" (the technical term for the light degrees of freedom) against the heavy quark. An equivalent picture in the combined limit is that of the model of Refs. [4 12] in which the heavy baryons are treated as harmonic collective motions of an ordinary baryon against a heavy meson. The symmetry emerges quite simply from this harmonic oscillator picture: the contracted $O(8)$ algebra is the algebra of all harmonic oscillator creation and annihilation operators and all bilinears made from them.

The key to the analysis of Ref. [2] which enables this picture to emerge is a consistent power-counting scheme. Formally, since we are considering the combined large $N_{c}$ and heavy quark limits, it is useful to consider a single parameter which characterizes departures from this limit. Thus, we introduce:

$$
\lambda=\Lambda / m_{Q}, 1 / N_{c},
$$

where $\Lambda$ is a typical strong interaction scale and $m_{Q}$ is the mass of the heavy quark. The effective expansion parameter turns out not to be $\lambda$, but $\lambda^{1 / 2}$ [2]. The low-lying (collective) excitation energies of the heavy baryons are of order $\lambda^{1 / 2}$. This should be contrasted with excitation energies for internal excitation of the brown muck and the dissociation energy of the heavy baryon (into an ordinary baryon and a heavy meson) which are each of order $\lambda^{0}$ [2]. Thus, provided that $\lambda^{1 / 2}$ can be considered to be small, a scale separation exists between the low-lying collective states of the heavy baryons from all other excitations. This, in turn, implies that EFT methods can be applied to this problem.

We note at the outset, however, that in practice $\lambda^{1 / 2} \sim 3^{-1 / 2}$ is not a particularly small number so that the scale separation is not particularly large. This implies that one must work at relatively high order in order to get relatively imprecise results. Thus for example, even working at next-to-leading order one only expects relative accuracy nominally only of order $\lambda$. Clearly, the approach will be at best semi-quantitative. Moreover, the expansion will not converge for all observables. On phenomenological grounds it is clear that at best the approach will be useful for describing the ground state of the $\Lambda_{Q}$ and the doublet of the first excited orbital excitation. The second orbitally excited states (which have not been observed in either the $\Lambda_{b}$ or $\Lambda_{c}$ sectors) is either unbound or very near the threshold and clearly beyond the harmonic limit implicit in the effective field theory. The situation is reminiscent of the contracted $S U\left(2 N_{F}\right)$ symmetries seen for light baryons which emerge in the large $N_{c}$ limit [13 17. In that case the large $N_{c}$ limit predicts an infinite tower of low-lying baryons. However, only the nucleon and delta for $N_{f}=2$ (or the octet and decuplet for $N_{f}=3$ ) are well described in the real world.

The construction of the form of the effective Hamiltonian or Lagrangian for the system is very straightforward. Following the standard rules of effective field theory, once the low-energy degrees of freedom are identified, one may simply write down the most general Lagrangian built from them which is consistent with the underlying symmetries and includes all terms up to a fixed order in the power counting. Similarly, one could add external sources to the theory and impose power counting and thereby determine the from of currents. Of course, in order to compute observables one needs more than the form of the effective Lagrangian and effective operators - one needs values for the coefficients. These coefficients can either be obtained purely phenomenologically or by matching with the underlying theory.

In this paper we derive the form of the effective Hamiltonian at next-to-leading order (NLO) directly from the structure of QCD and the power-counting rules. We do this formally by directly making a change of variables to the collective degrees of freedom. This has the benefit of relating the coefficients in the effective theory directly to ground state expectation values of known QCD operators. In this derivation the structure of the Hilbert space plays an essential role. In particular, it is necessary to show that in the combined limit, the Hilbert space can be written as a product space of collective excitations of order $\lambda^{1 / 2}$ which may be interpreted as excitations of the brown muck moving coherently against the heavy quark, and non-collective excitation of order $\lambda^{0}$ which correspond to excitations of the brown muck itself. In addition, as we will show, the excitations of the two spaces are independent up to corrections of higher order in $\lambda$.

We also derive results about the effective theory of more phenomenological interest. One of the central achievements of this paper is to show that at NLO the dynamics depends on only two free parameters in the effective Hamiltonian beyond known masses of the nucleon and heavy meson. This is of phenomenological significance in that the small value of the expansion parameter suggests that even for quite crude calculations one should work at least at NLO. Had there been a large number of free parameters at this order it would have suggested that the scheme would be difficult 
to implement in practice as data on heavy baryons becomes available. Another important result of this paper is the demonstration that certain operators that are not contained in the Hamiltonian but which play a role in describing electroweak properties of heavy baryons are completely determined without the need for phenomenological coefficients (up to corrections of higher order in $\lambda^{1 / 2}$ ) from the known structure of QCD and the counting rules. This increases the predictive power of the effective theory by eliminating free parameters.

As noted above, an expansion in $\lambda^{1 / 2}$ is unlikely to be rapidly convergent. Thus it seems sensible to work at relatively high order in the expansion. Here we have stopped at NLO for two reasons: one practical and one theoretical. The practical reason is simply that beyond NLO the number of parameters grows to the point where it is likely to exceed the number of observables which have any reasonable hope of being measured in the foreseeable future. The theoretical reason is that the change of variables technique used to derive the effective theory in terms of known operators in QCD is straightforward only up to NLO. Beyond this order, ambiguities in the definition of the heavy quark mass enter as do effects from the coupling of the low-lowing states described by the effective theory with higher energy excitations. Thus, the treatment becomes more subtle and complex beyond this order. Of course, this does not mean that there is no valid effective theory beyond NLO. However considerable effort must be undertaken in going beyond this order and this effort is simply not justified in view of the practical difficulties mentioned above.

This paper is organized as follows. In Sec. [I] we follow Ref. [2] and briefly review the derivation of the low energy collective variables for heavy baryons from QCD with an emphasis on the power counting rules. In Sec. III, we show that the structure of the Hilbert space as a product space emerges from standard large $N_{c}$ analysis. The form of the effective Hamiltonian (up to NLO) is derived from QCD in Sec. IV. Effective operators for electroweak matrix elements are derived in Sec. V. Finally a brief summary is given in Sec. VI

\section{DYNAMICAL VARIABLES FROM COUNTING RULES AND QCD}

In this section we review the counting rules derived from QCD for heavy baryons in the combined large $N_{c}$ and heavy quark limits. We will not reproduce the derivations of Ref. [2] in detail. Rather, we will outline the basic strategy of the derivation along with the principal physical and mathematical assumptions. We also collect results which will be needed later in this paper.

The first physical point of significance is to restrict our attention to a limited part of the QCD Hilbert space - the part with baryon number one and heavy quark number one. Furthermore we will ultimately restrict our attention to the lowest-lying states with these quantum numbers. For simplicity, in this section we will only consider a single species of heavy quark at a time as the strong interaction does not couple different heavy flavors. We will generalize to two heavy flavors when we consider electroweak matrix elements in the following sections.

QCD is a field theory and as such has an infinite number of degrees of freedom. We can envision making a canonical transformation from the original quark and gluon degrees of freedom to a new set of variables (at the quantum mechanical level it becomes a unitary transformation). The goal is to find such a transformation which has the property that a certain set of the variables (which we denote the collective variables), generate the low-lying collective excited states when acting (repeatedly) on the ground state (the $\Lambda_{Q}$ ). Working explicitly with the collective variables one can calculate directly the properties of the low-lying states. Because we are working in the context of canonical transformations it is far more natural to work with the Hamiltonian formulation of the underlying quantum field theory (QCD) rather than the Lagrangian formulation.

Unfortunately, there is no straightforward way to generate such a canonical transformation to collective variables exactly. However, it is easy to find a set of variables which behaves this way approximately. That is, using the powercounting scheme of Eq. (1.1) one can find collective variables which when acting on the ground state (repeatedly) generate the low-lying collective states plus a small component of higher excited states - the amount of higher excited state admixed is suppressed by powers of $\lambda^{1 / 2}$.

One canonically conjugate pair of collective variables is the total momentum of the system $\vec{P}$, the generator of translations (which is well defined in QCD) and its conjugate variable $\vec{X}$, the generator of momentum boosts. This pair of collective variables, however, does not induce the internal excitations of interest here.

One might also wish to introduce the momentum of the heavy quark as a collective variable. The expression for $\vec{P}_{Q}$ appropriate in the heavy quark limit is

$$
\vec{P}_{Q}=\int d^{3} x Q^{\dagger}(x)(-i \vec{D}) Q(x)
$$

where $Q(x)$ is the heavy quark field, and $\vec{D}$ is the three-dimensional covariant derivative. In the heavy quark limit it is apparent that the variable conjugate to $P_{Q}$ is $X_{Q}$ defined by 


$$
\vec{X}_{Q}=\int d^{3} x Q^{\dagger}(x) \vec{x} Q(x) .
$$

The definitions for $\vec{P}_{Q}$ and $\vec{X}_{Q}$ in Eqs. (2.1), (2.2) are clearly valid in the heavy quark limit for the subspace of states with a heavy quark number of unity; in this limit the heavy quark behaves as in nonrelativistic quantum mechanics. In the limit $\vec{P}_{Q}$ and $\vec{X}_{Q}$ correspond to the generators of translations and momentum boosts for the heavy quark. Away from this limit the concept of boosting or translating the heavy quark separately from the full system is not strictly well defined and the $\vec{P}_{Q}$ and $\vec{X}_{Q}$ defined above need not be canonically conjugate. However, even away from the formal limit there will exist some canonically conjugate pair of operators which smoothly goes to the variables defined in Eqs. (2.1), (2.2) as the limit is approached. Moreover, by standard heavy quark counting considerations this canonically conjugate pair of operators will differ from those defined in Eqs. (2.1), (2.2) by an amount of order $\mathcal{O}\left(\Lambda / m_{Q}\right)=\mathcal{O}(\lambda)$. Here we are interested in the theory up to next-to-leading order, i.e. up to relative order $\lambda^{1 / 2}$. Accordingly, to the order at which we work we can take Eqs. (2.1), 2.2) as unambiguous definitions.

Unfortunately, the conjugate pairs $(\vec{X}, \vec{P})$ and $\left(\vec{X}_{Q}, \vec{P}_{Q}\right)$ are not independent. Clearly $\left[\vec{P}, \vec{X}_{Q}\right] \neq 0$ since $\vec{P}$ is a generator of translations and translating the system necessarily translates the position of the heavy quark. Thus, we can not directly use $\left(\vec{X}_{Q}, \vec{P}_{Q}\right)$ as our collective variables to generate low-lying states. However, from these two pairs we can generate two independent conjugate pairs. In particular we can construct a linear combination of $\vec{P}$ and $\vec{P}_{Q}$, which we denote $\vec{p}$ and a linear combination of $\vec{X}$ and $\vec{X}_{Q}$, which we denote $\vec{x}$, such that $\vec{x}$ and $\vec{p}$ are conjugate to each other and commute with $\vec{P}$ and $\vec{X}$ up to corrections at next-to-next-to-leading order (NNLO). Intuitively $\vec{p}$ can be thought of as the generator of translations of the brown muck relative to the heavy quark and $\vec{x}$ as its conjugate. As we will see below, the variables $(\vec{x}, \vec{p})$ do act as approximate collective variables in the sense outlined above.

The explicit construction of $\vec{p}$ and $\vec{x}$ is detailed in Ref. [2]. The key inputs to this construction are Poincare invariance, heavy quark effective theory, and a decomposition of the QCD Hamiltonian organized via power-counting. The decomposition of the QCD Hamiltonian is most easily accomplished by thinking of the heavy baryon as a bound state of a heavy meson and a nucleon. Standard large $N_{c}$ QCD analysis allows us to identify the interaction energy as scaling as $N_{c}^{0}$, while the nucleon mass $m_{N}$ scales as $N_{c}$ [18,19]. Standard heavy quark analysis implies that the interaction energy is of order $m_{Q}^{0}$ while the mass of the heavy meson, $m_{H}$, is given by

$$
m_{H}=m_{Q}+\mathcal{O}\left(m_{Q}^{0}\right) .
$$

Thus, when constrained to the heavy baryon part of the Hilbert space, the QCD Hamiltonian may be decomposed as:

$$
\mathcal{H}=m_{H}+m_{N}+\mathcal{H}_{i n t},
$$

where $\mathcal{H}_{\text {int }}$ is of order $\lambda^{0}$. Poincare invariance implies that

$$
\left[X_{j}, \mathcal{H}\right]=i \frac{P_{j}}{\mathcal{H}},
$$

while the standard heavy quark treatment implies that

$$
\left[X_{Q_{j}}, \mathcal{H}\right]=i \frac{P_{Q_{j}}}{\mathcal{H}} .
$$

Using Eqs. 2.4), (2.5), (2.6) along with Eq.(2.3) and the fact that $m_{N}, m_{H} \sim \lambda$, the methods of Ref. [2] allows one to deduce that

$$
\begin{aligned}
& \vec{p}=\left(\frac{m_{H}}{m_{H}+m_{N}} \vec{P}-\vec{P}_{Q}\right)(1+\mathcal{O}(\lambda)), \\
& \vec{x}=\left(\left(1+\frac{m_{H}}{m_{N}}\right)\left(\vec{X}-\vec{X}_{Q}\right)\right)(1+\mathcal{O}(\lambda)) .
\end{aligned}
$$

The expressions for $\vec{x}$ and $\vec{p}$ differ from the equivalent expressions in Ref. [2] in that we have exploited Eq. (2.3) to express quantities in terms of $m_{H}$ which is directly physically accessible rather than $m_{Q}$ which is not. Throughout the remainder of this paper we will often eliminate $m_{Q}$ in favor of $m_{H}$.

Thus, we have constructed two independent pairs of conjugate variables: $(\vec{x}, \vec{p})$ and $(\vec{X}, \vec{P})$. These two pairs naturally arise in the Hamiltonian. However, as we will see in Sec. V] in describing electroweak operators these pairs are not so natural. Instead it is natural to use the positions and momenta of the heavy quarks $\left(\vec{X}_{Q}, \vec{P}_{Q}\right)$ and the 
corresponding independent set $\left(\vec{X}_{\ell}, \vec{P}_{\ell}\right)$ which correspond intuitively to the position and momenta of the light degrees of freedom. Formally they are defined as follows:

$$
\begin{aligned}
\vec{X}_{\ell} & =\vec{x}+\vec{X}_{Q}, \\
\vec{P}_{\ell} & =\vec{P}-\vec{P}_{Q} .
\end{aligned}
$$

In order to exploit the collective variables $\vec{x}$ and $\vec{p}$ to learn about the low-lying spectrum, it is useful to evaluate multiple commutators of $\vec{x}$ and $\vec{p}$ with $\mathcal{H}$. Following Ref. [2] one can again use Eqs. (2.3), (2.4), (2.5), (2.6) and the known $\lambda$ scaling of $m_{N}$ and $m_{H}$, i.e. $m_{N}, m_{H} \sim \lambda$, to obtain

$$
\left[x_{k},\left[x_{j}, \mathcal{H}\right]\right]=-\delta_{i j} / \mu(1+\mathcal{O}(\lambda)),
$$

where the reduced mass, $\mu$, is given by

$$
\mu=\frac{m_{N} m_{H}}{m_{N}+m_{H}} .
$$

More generally, it can be seen by the same methods that multiple commutators of $\vec{x}$ with $\mathcal{H}$ are suppressed by multiple powers of $\lambda$. In particular, if one takes $n$ commutators of components of $\vec{x}$ with $\mathcal{H}$ with $n$ even, then the $n^{\text {th }}$ commutator scales as $\lambda^{n-1}$. For example:

$$
\begin{aligned}
{\left[x_{m},\left[x_{l},\left[x_{k},\left[x_{j}, \mathcal{H}\right]\right]\right]\right] } & \sim \lambda^{3}, \\
{\left[x_{o},\left[x_{n},\left[x_{m},\left[x_{l},\left[x_{k},\left[x_{j}, \mathcal{H}\right]\right]\right]\right]\right]\right] } & \sim \lambda^{5} \\
& \cdots
\end{aligned}
$$

In contrast, standard large $N_{c}$ counting implies that a displacement of the light degrees of freedom relative to the heavy degrees of freedom by a distance of order $\lambda^{0}$ shifts the Hamiltonian by an amount of order $\lambda^{0}$ :

$$
e^{-i \vec{p} \cdot \vec{y}} \mathcal{H} e^{i \vec{p} \cdot \vec{y}}-\mathcal{H} \sim \mathcal{O}\left(\lambda^{0}\right),
$$

where $\vec{y}$ is a c-number of order $\lambda^{0}$. Equation (2.12) in turn implies that multiple commutators of $\vec{p}$ with $\mathcal{H}$ are generically of order unity:

$$
\begin{aligned}
{\left[p_{k},\left[p_{j}, \mathcal{H}\right]\right] } & \sim \lambda^{0}, \\
{\left[p_{m},\left[p_{l},\left[p_{k},\left[p_{j}, \mathcal{H}\right]\right]\right]\right] } & \sim \lambda^{0}, \\
{\left[p_{o},\left[p_{n},\left[p_{m},\left[p_{l},\left[p_{k},\left[p_{j}, \mathcal{H}\right]\right]\right]\right]\right]\right] } & \sim \lambda^{0} \\
& \cdots
\end{aligned}
$$

The power-counting rules from QCD are essentially summarized in Eqs. (2.11) and (2.13). By themselves, however, they are not sufficient to develop a fully systematic power-counting scheme for observables without additional phenomenological input. In particular, there are two possible scenarios in which the power-counting may be realized selfconsistently. Intuitively, the $O(8)$ or symmetric realization corresponds to the case where the effective potential between the heavy quark and the brown muck has a minimum at $\vec{x}=0$, while the $O(4) \otimes O(4)$ or symmetry broken realization corresponds to the situation where the effective potential has a minimum at $\vec{x} \neq 0$. The $O(4) \otimes O(4)$ realization is labeled symmetry broken since it breaks rotational symmetry in the $\lambda \rightarrow 0$ limit. As shown in Ref. [2] the $O(8)$ (symmetric) realization has the generic property that

$$
x \sim \lambda^{1 / 4}, p \sim \lambda^{-1 / 4}
$$

in the sense that typical matrix elements between low-lying states of polynomial operators which contain $x^{n_{x}}$ and $p^{n_{p}}$ scale as $\lambda^{\left(n_{x}-n_{p}\right) / 4}$. In contrast, in the $O(4) \otimes O(4)$ realization,

$$
x \sim \lambda^{0}, p \sim \lambda^{-1 / 4} .
$$

In this paper we will assume that the dynamics are such that the system is in the $O(8)$ (symmetric) realization. 


\section{STRUCTURE OF THE HILBERT SPACE}

In this section we will show that in the combined large $N_{c}$ and heavy quark limits, the Hilbert space can be written as a product space composed of collective and intrinsic excitations, with the collective excitations having energies of order $\lambda^{1 / 2}$ and intrinsic excitations are of order unity. The physical picture of these two types of excitations is quite clear - the collective excitations describe coherent motion of the brown muck against the heavy quark while the intrinsic excitations are excitations of the brown muck itself.

The basic strategy in demonstrating that the Hilbert space is of this form is to exploit the fact that our expansion parameter is valid in the pure large $N_{c}$ limit where Witten's Hartree picture is well established [19]. The key point is that while nature is far closer to the heavy quark limit than the large $N_{c}$ limit, our expansion is formulated in the combined large $N_{c}$ and heavy quark limits for $N_{c} \Lambda / m_{Q}$ arbitrary. Thus it applies whether one takes the heavy quark limit first, the large $N_{c}$ limit first or takes the limits simultaneously. We will deduce the structure of the Hilbert space by taking the large $N_{c}$ limit first and then argue that the result goes over smoothly to the combined limit. Ultimately, the reason that we can legitimately take the limits in any order stems from the fact that the dynamics of the system are determined by the interaction Hamiltonian, $\mathcal{H}_{\text {int }}=\mathcal{H}-m_{H}-m_{N}$. Note that $\mathcal{H}_{\text {int }}$ is non-singular in both the large $N_{c}$ and heavy quark limits, and therefore we can smoothly take either limit or the combined limit first.

Now consider taking the large $N_{c}$ limit prior to taking the heavy quark limit. In this case we can use Witten's Hartree picture of baryons. Each quark propagates in the mean field of the remaining quarks. Low energy excitations are described by the excitations of a single quark in the background of the remaining quarks. In the present case we can divide these excitations into two classes - excitations of the heavy quark and excitations of one of the light quarks. Of course, for the light quark excitation it is necessary to anti-symmetrize the wave function as there are many light quarks. In contrast there is only one heavy quark. In the large $N_{c}$ limit these two classes of excitations are orthogonal to each other so that the wave function may be written as a direct product:

$$
|\psi\rangle_{\mathrm{N}_{\mathrm{c}} \rightarrow \infty}=|h\rangle \otimes|\ell\rangle
$$

where $h(\ell)$ represent the heavy (light) quark part of the Hartree wave function.

The excitation energies for both types of excitations are of order $N_{c}^{0}$. However, the excitation energies of the heavy quark are of order $m_{Q}^{-1 / 2}$. This is straightforward to see; the heavy quark sits in a potential well of depth and width independent of $m_{Q}$. As one takes the large $m_{Q}$ limit (after having taken the large $N_{c}$ limit), the heavy quark wave function becomes localized at the bottom of the potential well and thus acts like an harmonic oscillator. One sees that the characteristic excitation energies of the heavy and light types of motion scale with $\lambda$ according to

$$
\begin{aligned}
& \Delta E_{h} \sim N_{c}^{0} m_{Q}^{-1 / 2} \sim \lambda^{1 / 2}, \\
& \Delta E_{\ell} \sim N_{c}^{0} m_{Q}^{0} \sim \lambda^{0} .
\end{aligned}
$$

As noted above, in this picture the excitations of the heavy quark and the light quarks are independent. The total excitation energy is simply the sum of the heavy excitation energy and the light excitation energy.

If we now consider the combined limit with the large $N_{c}$ and heavy quark limits taken simultaneously the picture changes. The Hartree approximation is no longer valid. However, the general counting rules remain — they simply have to be reinterpreted. Physically what happens in the combined limit is that instead of the heavy quark oscillating in the static field of the brown muck, as in the Hartree picture, they oscillate against each other. However, the structure of the Hilbert space is not altered in its essence. The space can still be broken up into a product space of independent classes of excitations as in Eq. (3.1), but now the two classes of excitations are not the heavy and light degrees of freedom as in the Hartree approximation. Rather, they are the collective excitations (between the brown muck and the heavy quark) and the intrinsic excitations (of the brown muck):

$$
|\psi\rangle_{\lambda \rightarrow 0}=|\mathrm{C}\rangle \otimes|\mathrm{I}\rangle
$$

where C ( I ) represent the collective (intrinsic) part. Similarly, the excitation energies behave as in Eq. (3.2) but with the more general description of collective and intrinsic classes of excitations replacing the heavy and light classes of the Hartree approximation:

$$
\begin{aligned}
\Delta E_{C} & \sim \lambda^{1 / 2} \\
\Delta E_{I} & \sim \lambda^{0} .
\end{aligned}
$$

We see that the structure of the Hilbert space is quite simple in the $\lambda \rightarrow 0$ limit. In order to understand the structure of the effective theory, it is necessary to understand how corrections to this leading behavior emerge. In 
particular, we will need to quantify the mixing between the collective and intrinsic excitations due to sub-leading terms in $\lambda$. Again, our strategy is to pass to the case where the large $N_{c}$ limit is taken first and we have the Hartree approximation as a well-defined tool. Then use the smoothness of the two limits to pull back to the case of $\lambda \rightarrow 0$ keeping $N_{c} \Lambda / m_{Q}$ arbitrary.

It is useful to consider the way $1 / N_{c}$ corrections emerge on top of the Hartree picture as these corrections will ultimately become corrections in our $\lambda$ counting. We begin by assuming that at finite, but large $N_{c}$, a Hartree picture is a good first approximation. We also make the standard large $N_{c}$ assumption [19] that corrections to the energy and to energy splittings are generically of relative order $1 / N_{c}$. Moreover, these leading relative corrections are independent of $m_{Q}$ as the heavy quark only contributes 1 out of $N_{c}$ quarks. Thus, these relative corrections due to effects beyond the Hartree approximation are necessarily of order $\lambda$ and do not contribute at NLO (which is relative order $\lambda^{1 / 2}$ ). It is straightforward to see that $1 / N_{c}$ corrections to the energies imply that Hamiltonian matrix elements between two different states with different intrinsic states are generically of order $N_{c}^{-1 / 2}$. This can be shown in two ways. First, off-diagonal matrix elements between different intrinsic states of order $N_{c}^{-1 / 2}$ yield $N_{c}^{-1}$ corrections to the energies and we know corrections of this order exist. The second way is to construct an explicit wave function using a transition induced by the gluon exchange. The gluon exchange contains two factors of the strong coupling and scales as $N_{c}^{-1}$; there is a combinatoric factor of $N_{c}$ as the gluon can connect to any quark; and finally, there is a normalization factor of $N_{c}^{-1 / 2}$ because there are $N_{c}$ distinct terms in the excited state wave function as any of the quarks may be excited. Now we can pass from the large $N_{c}$ limit back to the combined $\lambda \rightarrow 0$ limit (with $N_{c} \Lambda / m_{Q}$ fixed) by exploiting smoothness. We obtain the following important result:

$$
\left\langle\mathrm{C}, \mathrm{I}|\mathcal{H}| \mathrm{C}, \mathrm{I}^{\prime}\right\rangle \sim \lambda^{1 / 2},
$$

where the state $|\mathrm{C}, \mathrm{I}\rangle$ is a product state

$$
|\mathrm{C}, \mathrm{I}\rangle=|\mathrm{C}\rangle \otimes|\mathrm{I}\rangle .
$$

\section{CONSTRUCTION OF THE EFFECTIVE HAMILTONIAN}

In Sec. II we reviewed the power-counting rules for the collective variables $\vec{x}$ and $\vec{p}$ in the heavy baryon sector of the QCD Hilbert space. As discussed in Sec. I, the general principle of effective field theory implies that the form of the effective theory is completely fixed by the symmetries of the theory and the power-counting rules. Rather than simply invoking this general strategy, it is instructive to derive the effective field theory at next-to-leading order directly from QCD through the change in variables discussed in Sec. II. We do this because it is useful to verify that the proposed scaling rules are, indeed, selfconsistent; the derivation gives significant insights into the underlying structure of the theory; and finally, it is satisfying to verify explicitly the validity of the general EFT philosophy in this case.

In the present context, where we have been implementing canonical transformations to collective variables, it is simpler to work with an effective Hamiltonian rather than an effective Lagrangian. From Eq. (2.5) and the powercounting rules of Eqs. (2.9), (2.11), (2.13) and (2.14) it is straightforward to deduce the form of the QCD Hamiltonian in the heavy baryon sector written in terms of the collective variables up to order $\lambda$ (NLO). It is given by

$$
\begin{aligned}
\mathcal{H}_{\mathrm{QCD}} & =m_{H}+m_{N}+\frac{P^{2}}{2\left(m_{N}+m_{H}\right)}+\frac{p^{2}}{2 \mu}+\hat{c}^{(0)}+\sum_{j} \hat{c}_{j}^{(1)} x_{j}+\sum_{j, k} \hat{c}_{j, k}^{(2)} x_{j} x_{k} \\
& +\sum_{j, k, l} \hat{c}_{j, k, l}^{(3)} x_{i} x_{j} x_{l}+\sum_{j, k, l, m} \hat{c}_{j, k, l, m}^{(4)} x_{i} x_{j} x_{k} x_{l}+\mathcal{O}\left(\lambda^{3 / 2}\right),
\end{aligned}
$$

where $m_{H}, m_{N} \sim \lambda^{-1}$, and $\hat{c}^{(a)} \sim \lambda^{0}$. From rotational invariance, the $\hat{c}^{(a)}$ are completely symmetric Cartesian tensor operators of rank $a$. Note that while terms up to $x^{4}$ contribute at this order, only terms up to $p^{2}$ contribute. The difference between these two is due to the essentially nonrelativistic nature of the kinematics. Formally, this difference is implicit in the different scaling relations for $x$ and $p$ in Eqs. (2.9), (2.11), 2.13) and (2.14).

Since the form of Eq. (4.1) was deduced from commutation relations, one cannot immediately conclude that the $\hat{c}^{(a)}$ operators are c-numbers. Rather, at this stage in the derivation we can only deduce that they are operators which commute with all of the collective variables:

$$
\left[\vec{x}, \hat{c}^{(a)}\right]=\left[\vec{X}, \hat{c}^{(a)}\right]=\left[\vec{p}, \hat{c}^{(a)}\right]=\left[\vec{P}, \hat{c}^{(a)}\right]=0 .
$$


Thus, the $\hat{c}^{(a)}$ tensors are, in general, operators which depend only on the non-collective variables, i.e. the $\hat{c}^{(a)}$ are purely intrinsic operators. Note that to the extent that the coefficients $\hat{c}^{(a)}$ in the Hamiltonian of Eq. (4.1) are operators as opposed to c-numbers, the Hamiltonian is not strictly an effective Hamiltonian. Rather it is the full QCD Hamiltonian written in terms of our collective degrees of freedom. The non-collective degrees of freedom have not been integrated out; rather their effects are contained in the non-collective operators $\hat{c}^{(a)}$.

Non-collective operators might be expected to influence the dynamics of the collective degrees of freedom even though they all commute with the collective degrees of freedom. For example, consider the ground state matrix element of $\left(\hat{c}_{i}^{(1)} x_{i}\right)^{2}$. This can be expressed in terms of off-diagonal matrix elements:

$$
\left\langle 0\left|\left(\hat{c}_{i}^{(1)} x_{i}\right)\left(\hat{c}_{i}^{(1)} x_{i}\right)\right| 0\right\rangle=\sum_{\alpha_{C}}\left\langle 0\left|\left(\hat{c}_{i}^{(1)} x_{i}\right)\right| \alpha_{C}\right\rangle\left\langle\alpha_{C}\left|\left(\hat{c}_{i}^{(1)} x_{i}\right)\right| 0\right\rangle+\sum_{\beta_{N C}}\left\langle 0\left|\left(\hat{c}_{i}^{(1)} x_{i}\right)\right| \beta_{N C}\right\rangle\left\langle\beta_{N C}\left|\left(\hat{c}_{i}^{(1)} x_{i}\right)\right| 0\right\rangle,
$$

where $|0\rangle$ is the ground state of the heavy baryon system and the intermediate states are grouped into purely collective excitations $\left|\alpha_{C}\right\rangle$ (with excitation energies of order $\lambda^{1 / 2}$ ) which excite only the collective degrees of freedom leaving the brown muck unexcited, and non-collective states $\left|\beta_{N C}\right\rangle$ (with excitation energies of order $\lambda^{0}$ or greater) which involve an intrinsic excitation of the brown muck. If the operators in the Hamiltonian had been purely collective as in a true effective Hamiltonian (which by construction has all non-collective degrees of freedom integrated out), then the sum would not contain the non-collective states $\left|\beta_{N C}\right\rangle$. However, the non-collective operators $\hat{c}^{(a)}$ induce transitions to the non-collective states. By acting twice - once to go into the non-collective space, and once to come back the collective space - the non-collective operators can affect collective space matrix elements of operators contained in the Hamiltonian.

However, from Eq. (3.5) we know that terms which connect different intrinsic states are suppressed by order $\lambda^{1 / 2}$. We can use this to deduce that the contribution of non-collective intermediate states to matrix elements of composite operators such as in Eq. (4.3) will always be suppressed by order $\lambda$ relative to the contributions of the collective intermediated states and thus only contribute at NNLO. This in turn justifies their omission at NLO. Let us see how this works for the decomposition in Eq. (4.3). From the scaling rules of Eq. (2.14) it is straightforward to see that the contribution from the collective intermediate states goes as $\lambda^{1 / 2}$. A typical term in the sum over states outside the purely collective space is

$$
\left|\left\langle 0_{\mathrm{C}}, 0_{\mathrm{I}}\left|c_{i}^{(1)} x_{i}\right| \mathrm{C}, \mathrm{I}\right\rangle\right|^{2}=\left|\left\langle 0_{\mathrm{C}}\left|x_{i}\right| \mathrm{C}\right\rangle\right|^{2}\left|\left\langle 0_{\mathrm{I}}\left|c_{i}^{(1)}\right| \mathrm{I}\right\rangle\right|^{2} \sim \lambda^{1 / 2} \lambda,
$$

where $\left|0_{\mathrm{C}}\right\rangle$ and $\left|0_{\mathrm{I}}\right\rangle$ represent the ground states of the collective and intrinsic subspaces, respectively, while $|\mathrm{C}\rangle$ and $|\mathrm{I}\rangle$ represent excited states, and the notation $|\mathrm{C}, \mathrm{I}\rangle$ represents a product state as in Eq. (3.6). The equality in Eq. (4.4) follows since $x_{i}$ only acts on the collective space while $c_{i}^{(1)}$ only acts on the intrinsic space and the scaling behavior follows from Eqs. (2.14) and (3.5) so that the collective part of the expectation value scales as $\lambda^{1 / 2}$ while the intrinsic part scales as $\lambda$. Thus, we see that the contribution of non-collective states is down by a factor of $\lambda$ compared to the contribution of the collective intermediate states.

The $\mathcal{O}(\lambda)$ suppression of the effects of non-collective intermediate states relative to the collective ones is generic. Although it was explicitly demonstrated for the case of the composite operator in Eq. (4.3), it should be apparent that such a suppression occurs for all products of operators contained in the Hamiltonian. When calculating Hamiltonian matrix elements, the effects of the operators $\hat{c}^{(a)}$ in Eq. (4.1) inducing mixing with the non-collective states will always contribute at relative order $\lambda$ (i.e. at NNLO) or less. Thus, working at NLO one can simply replace the $\hat{c}^{(a)}$ operators by their expectation values in the ground state (or more generally in the ground state band - the set of collective states built on the ground state of the intrinsic degrees of freedom). Therefore, the operators $\hat{c}^{(a)}$ can be treated as c-numbers in the effective theory.

When the coefficients $\hat{c}^{(a)}$ are treated as operators, rotational invariance does not constrain the allowable forms in the Hamiltonian beyond what is given in Eq. (4.1). For example, the term $\sum_{i} \hat{c}_{i}^{(1)} x_{i}$ is allowable despite the fact that $\vec{x}$ is an $L=1$ operator; $\hat{c}^{(1)}$ could also be $L=1$ operator and couple to $\vec{x}$ leading to an $L=0$ operator in the Hamiltonian. Once the $\hat{c}^{(a)}$ operators are restricted to being c-numbers in the effective theory, rotational invariance greatly restricts the allowable forms: c-numbers do not transform under spatial rotations. Accordingly, the only surviving $\hat{c}^{(a)}$ coefficients are those which multiply rotational scalars. Thus, for example, the $\hat{c}^{(1)}$ terms must vanish. Treating the $\hat{c}^{(a)}$ coefficients as c-numbers and imposing rotational symmetry yields the following form of the effective Hamiltonian up to $\mathcal{O}(\lambda)$ :

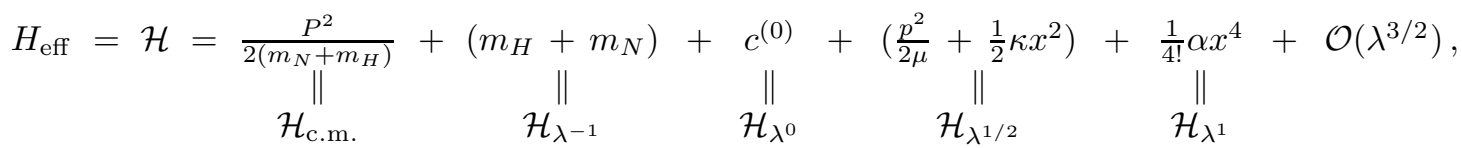


where $\mathcal{H}_{c . m}$. refers to the center of mass motion of the entire system while $\mathcal{H}_{\lambda^{n}}$ refers to the piece of the Hamiltonian whose leading contribution is of order $\lambda^{n}$. The coefficients $c^{(0)}, \kappa$ and $\alpha$ are of order $\lambda^{0}$ and may be expressed as ground-state expectation values of $\hat{c}^{(0)}, \hat{c}^{(2)}$ and $\hat{c}^{(4)}$, respectively:

$$
\begin{aligned}
c^{(0)} & =\left\langle 0\left|\hat{c}^{(0)}\right| 0\right\rangle, \\
\kappa & =\frac{2}{3} \sum_{i}\left\langle 0\left|\hat{c}_{i, i}^{(2)}\right| 0\right\rangle, \\
\alpha & =\frac{24}{5} \sum_{i, j}\left\langle 0\left|\hat{c}_{i, i, j, j}^{(4)}\right| 0\right\rangle .
\end{aligned}
$$

A few comments about this derivation are in order here. In essence, we did not have to explicitly integrate out the non-collective higher-lying degrees of freedom. By making a change of variables to $\vec{x}, \vec{p}, \vec{X}$ and $\vec{P}$, we already have identified variables which were decoupled from the intrinsic degrees of freedom to the order at which we are working. Imagine one explicitly made this change of variables prior to formulating the problem in terms of a functional integral. At low order the action could be written as the sum of a collective action plus an intrinsic action without coupling between them, and the only effect of integrating out the intrinsic degrees of freedom would be to supply an overall constant multiplying the remaining functional integral over the collective degrees of freedom. By making this change of variables we make the effect of integrating out the intrinsic degrees of freedom trivial - at least up to NLO. Only if we go beyond this order do effects of the the coupling to the intrinsic degrees of freedom play a role, and the effects of integrating out the intrinsic degrees of freedom become nontrivial.

From a phenomenological prospective, it is sufficient to start with Eq. (4.5). The coefficients $c^{(0)}, \kappa$ and $\alpha$ could then be determined approximately from fits to experimental data. Given the fact that the masses of the nucleon and the heavy meson are known, the effective theory at NLO depends on three parameters $-c^{(0)}, \kappa$ and $\alpha$. The coefficient $c^{(0)}$ is determined from the difference in energy between the nucleon-heavy meson threshold and the lowest energy state. However, $c^{(0)}$ is simply an overall constant and plays no role in the dynamics; the dynamics of the problem depends on two parameters at NLO. It is worth noting that at leading order the dynamics depends only on a single parameter, $\kappa$. Thus, going from leading order to next-to-leading costs only an additional parameter, $\alpha$.

\section{EFFECTIVE OPERATORS}

To make predictions of electroweak current matrix elements between heavy baryon states, one needs more than just the effective Hamiltonian. Effective operators are also required. For general composite operators expressed in terms of the quark and glue degrees of freedom of QCD, the effective operators can be derived in a functional integral form by integrating out the intrinsic degrees of freedom. The most straightforward way to formulate this is as an integral over all QCD variables with $\delta$-functions put in to pick out particular values of the collective variables. In practice, however, the preceding description is rather formal as it is not generally possible to perform this functional integration. At a phenomenological level, one can simply write a form for the operator consistent with symmetries and parameterized by some constants and then fit the constants from experimental data. However, if the operators of interest can be expressed entirely in terms of the collective degrees of freedom at the QCD level, the functional integral approach becomes unnecessary (for the same reason it was unnecessary in the derivation of the collective Hamiltonian). At NLO, one can immediately express the effective operators in terms of the same collective degrees of freedom.

To see how this works, consider some operator which at the QCD level can be expressed entirely in terms of the collective operators. We seek to find an analogous operator for use in the effective theory whose matrix elements in the effective theory reproduces the matrix elements of the QCD operator in the QCD states (up to correction at NNLO). Let us start by considering the matrix elements at the QCD level. From the discussion of the structure of the Hilbert space at the QCD level in Sec. III, it is apparent that a low-energy (i.e. with excitation energy of order $\lambda^{1 / 2}$ ) heavy baryon state in a $\lambda^{1 / 2}$ expansion at NLO can be written as:

$$
|\psi\rangle=\left|\mathrm{C}, 0_{\mathrm{I}}\right\rangle+\lambda^{1 / 2} \sum_{\mathrm{C}^{\prime}, \mathrm{I} \neq 0_{\mathrm{I}}} a_{\mathrm{C}^{\prime}, \mathrm{I}}\left|\mathrm{C}^{\prime}, \mathrm{I}\right\rangle,
$$

where $|\mathrm{C}, \mathrm{I}\rangle$ is defined as a product state as in Eq. (3.6), and $a_{\mathrm{C}^{\prime}, \mathrm{I}}$ are constants of order unity.

The sum in the second term of Eq. (5.1) is restricted to contributions with $I \neq 0_{\text {I }}$ due to the product structure of the state. If the sum were not restricted, then there would be contributions of the form 


$$
\begin{aligned}
& \left|\mathrm{C}, 0_{\mathrm{I}}\right\rangle+\lambda^{1 / 2} \sum_{\mathrm{C}^{\prime}} a_{\mathrm{C}^{\prime}, 0_{\mathrm{I}}}\left|\mathrm{C}^{\prime}, 0_{\mathrm{I}}\right\rangle=|\mathrm{C}\rangle \otimes\left|0_{\mathrm{I}}\right\rangle+\lambda^{1 / 2} \sum_{\mathrm{C}^{\prime}} a_{\mathrm{C}^{\prime}, 0_{\mathrm{I}}}\left|\mathrm{C}^{\prime}\right\rangle \otimes\left|0_{\mathrm{I}}\right\rangle \\
& =\left(|\mathrm{C}\rangle+\lambda^{1 / 2} \sum_{\mathrm{C}^{\prime}} a_{\mathrm{C}^{\prime}, 0_{\mathrm{I}}}\left|\mathrm{C}^{\prime}\right\rangle\right) \otimes\left|0_{\mathrm{I}}\right\rangle=\left|\tilde{\mathrm{C}}, 0_{\mathrm{I}}\right\rangle
\end{aligned}
$$

where, $|\tilde{\mathrm{C}}\rangle=\left(|\mathrm{C}\rangle+\lambda^{1 / 2} \sum_{\mathrm{C}^{\prime}} a_{\mathrm{C}^{\prime}, 0_{\mathrm{I}}}\left|\mathrm{C}^{\prime}\right\rangle\right)$ is simply a single state in the purely collective space. Thus, the contribution to the sum from Eq. (5.2) is of the form of a single product state. If there are contributions of the form as in Eq. (5.2) we can replace $\mathrm{C}$ by $\mathrm{C}$ and thereby eliminate contributions with $\mathrm{I}=0_{\mathrm{I}}$ from the sum. Doing this has the advantage of removing any possibility of double counting these contributions.

Now let us evaluate the matrix element of a purely collective operator, $\Theta_{\mathrm{C}}$, i.e. an operator constructed entirely of the collective operators $\vec{x}, \vec{p}, \vec{X}$ and $\vec{P}$. From the structure of the state in Eq. (5.1), the matrix element between two low-energy heavy baryon states (given at NLO) is given by

$$
\begin{aligned}
& \left\langle\psi_{1}\left|\Theta_{\mathrm{C}}\right| \psi_{2}\right\rangle=\left(\left\langle\mathrm{C}_{1}, 0_{\mathrm{I}_{1}}\left|+\lambda^{1 / 2} \sum_{\mathrm{C}_{1^{\prime}, \mathrm{I}_{1} \neq 0_{\mathrm{I}_{1}}}} a_{\mathrm{C}_{1}, \mathrm{I}_{1}}^{*}\left\langle\mathrm{C}_{1}{ }^{\prime}, \mathrm{I}\right|\right) \Theta_{\mathrm{C}}\left(\left|\mathrm{C}_{2}, 0_{\mathrm{I}_{2}}\right\rangle+\lambda^{1 / 2} \sum_{\mathrm{C}_{2}{ }^{\prime}, \mathrm{I}_{2} \neq 0_{\mathrm{I}_{2}}} a_{\mathrm{C}_{2}{ }^{\prime}, \mathrm{I}_{2}}\left|\mathrm{C}_{2}{ }^{\prime}, \mathrm{I}_{2}\right\rangle\right)\right.\right. \\
& =\left\langle\mathrm{C}_{1}\left|\Theta_{\mathrm{C}}\right| \mathrm{C}_{2}\right\rangle+\lambda \sum_{\mathrm{C}_{1^{\prime}, \mathrm{C}_{2}{ }^{\prime}, \mathrm{I}_{1} \neq 0_{\mathrm{I}_{1}}, \mathrm{I}_{2} \neq 0_{\mathrm{I}_{2}}}} \delta_{\mathrm{I}_{1}, \mathrm{I}_{2}} a_{\mathrm{C}_{1}{ }^{\prime}, \mathrm{I}_{1}}^{*} a_{\mathrm{C}_{2}{ }^{\prime}, \mathrm{I}_{2}}\left\langle\mathrm{C}_{1}{ }^{\prime}\left|\Theta_{\mathrm{C}}\right| \mathrm{C}_{2}{ }^{\prime}\right\rangle=\left\langle\mathrm{C}_{1}\left|\Theta_{\mathrm{C}}\right| \mathrm{C}_{2}\right\rangle(1+\mathcal{O}(\lambda)) .
\end{aligned}
$$

Thus, up to NNLO corrections the QCD matrix element is simply the matrix element in the collective part of the Hilbert space. On the other hand, the effective Hamiltonian we use at this order with the operators $\hat{c}^{(a)}$ replaced by c-numbers is designed to correctly describe the collective part of the Hilbert space at this order.

The preceding argument shows, that in the case of purely collective operators the operators in the effective theory are related to the QCD operators in a trivial way up to corrections at NNLO. A purely collective operator at the QCD level is of the form

$$
\Theta_{\mathrm{C}}^{\mathrm{QCD}}\left(\vec{x}^{\mathrm{QCD}}, \vec{p}^{\mathrm{QCD}}, \vec{X}^{\mathrm{QCD}}, \vec{P}^{\mathrm{QCD}}\right)
$$

where the superscript QCD indicates that all of the operators are at the QCD level. The effective operator up to order $\lambda$ corrections is then obtained by keeping the same operator structure of $\Theta_{\mathrm{C}}^{\mathrm{QCD}}$, but replacing $\vec{x}^{\mathrm{QCD}}, \vec{p}^{\mathrm{QCD}}$, $\vec{X}^{\mathrm{QCD}}$ and $\vec{P}^{\mathrm{QCD}}$ with the collective operators $\vec{x}, \vec{p}, \vec{X}$ and $\vec{P}$ :

$$
\Theta^{\mathrm{eff}} \equiv \Theta_{\mathrm{C}}^{\mathrm{QCD}}(\vec{x}, \vec{p}, \vec{X}, \vec{P}) .
$$

This is essentially a nonrenormalization theorem to this order. The effects of integrating out collective degrees of freedom only renormalize the effective operators at NNLO.

In a similar way, all purely non-collective operators at the QCD level, i.e. all operators which commute with all of the collective operators, simply become c-numbers in the effective theory with a value equal to the ground state expectation value of the analogous QCD operator. These general principles can be used to deduce the form of several operators in the effective theory which will prove useful in the calculation of electroweak observables for heavy baryon states.

Let us first consider the operator which generates Lorentz boosts, $\vec{K}$. The unitary operator for a Lorentz boost is $B_{\vec{v}}=e^{i \vec{v} \cdot \vec{K}}$. The boost operator is useful, for example, in calculating form factors which involve matrix elements of states with different momenta. One can use the boost operator to generate these states from a standard state in its rest frame. From Poincare invariance, the boost operator is given at the field theoretic level by

$$
\vec{K}=\frac{1}{2}(\vec{X} \mathcal{H}+\mathcal{H} \vec{X})=\left(m_{N}+m_{H}\right) \vec{X}+\mathcal{O}(\lambda),
$$

where the second equality follows from Eq. (2.4). Thus, up to NLO at the QCD level $\vec{K}=\left(m_{N}+m_{H}\right) \vec{X}$ and from the arguments given above, this can immediately be taken over in the effective theory.

Among the observables which can be studied using the combined expansion are matrix elements of the electroweak current $J=\bar{Q}_{j} \Gamma Q_{i}$, where subscripts $i$ and $j$ refer to the heavy quark flavors and $\Gamma$ is the Dirac structure of the left-hand current, $\Gamma=\gamma^{\mu}\left(1-\gamma^{5}\right)$. We will show here that the leading term in the combined heavy quark and large $N_{c}$ expansion of the current $J$ can be expressed in terms of the collective variables $\vec{x}, \vec{p}, \vec{X}, \vec{P}$. Moreover, we will show that the lowest order corrections come at order $\lambda$ and not at $\lambda^{1 / 2}$, so that they contribute only at NNLO. 
The combined expansion of the current $J$ can be done analogously to the pure heavy quark expansion in the Heavy Quark Effective Theory (HQET) 20 25. The idea is to decompose the total heavy quark field into "large" and "small" components or "light" and "heavy" fields with the latter being suppressed by a factor of $1 / m_{Q}$ relative to the "large" component. It is then possible to eliminate the "small" component in terms of the "large" component by means of a power series expansion. Near the combined limit considered here, the pure heavy quark expansion breaks down. It has been shown in Refs. 26 29] that the next-to-leading order terms in the pure heavy quark expansion of the matrix elements of the current $J$ between the heavy baryon states contain coefficients proportional to $\bar{\Lambda} / m_{Q}$ where $\bar{\Lambda}=m_{\Lambda_{Q}}-m_{Q}$ (with $m_{\Lambda_{Q}}$ being the total mass of a heavy baryon). In the HQET the constant $\bar{\Lambda}$ is treated as being much smaller than $m_{Q}$, so that the NLO operators are indeed suppressed. The situation is different, however, near the combined limit where $\bar{\Lambda}$ is of order $m_{N}$, so that $\bar{\Lambda} \sim \lambda^{-1}$ and $\bar{\Lambda} / m_{Q} \sim \lambda^{0}$. Hence, these corrections cannot be neglected near the combined limit.

The appearance of large corrections near the combined limit is a result of the way the total heavy quark field is separated into its "large" and "small" components. The definition of these components is necessarily different from the analogous decomposition in HQET. Near the combined limit, as in HQET, the heavy quark is not far off-shell since its interaction with the brown muck is of order $\lambda^{0}$ while its mass is of order $\lambda^{-1}$. However, the space-time dependence of the heavy quark is much different near the combined limit. Near the pure heavy quark limit the brown muck mass is formally much less than the heavy quark mass. As a result, the space-time dependence of the heavy quark is essentially determined by the free particle Lagrangian. In HQET, therefore, it is useful to redefine the heavy quark field by removing the phase factor of the solution of the free Dirac equation, namely $e^{-i m_{Q} v^{\mu} y_{\mu}}$. Near the combined limit the heavy quark remains essentially nonrelativistic. However, the space-time dependence of the heavy quark is much different from that of a free particle. The phase factor now should contain a contribution due to the brown muck mass, which is formally of the same order as the heavy quark mass near the combined limit. This naturally leads to the following redefinition of the heavy quark field:

$$
\begin{aligned}
& h_{Q}^{(v)}(y)=e^{-i\left(m_{Q}+m_{N}\right) v^{\mu} y_{\mu}} P_{+} Q(y), \\
& H_{Q}^{(v)}(y)=e^{-i\left(m_{Q}+m_{N}\right) v^{\mu} y_{\mu}} P_{-} Q(y),
\end{aligned}
$$

where $v^{\mu}$ is the total 4 -velocity of the heavy baryon, the operators $P_{ \pm}=(1 \pm \not) / 2$ in the rest frame of the heavy baryon project out the upper and lower components of the heavy quark field, and $y$ here represents a space-time point. As in HQET, the fields $h_{Q}^{(v)}$ and $H_{Q}^{(v)}$ satisfy conditions $\not h_{Q}^{(v)}=h_{Q}^{(v)}$ and $\not 0 H_{Q}^{(v)}=-H_{Q}^{(v)}$. Fields $h_{Q}^{(v)}$ and $H_{Q}^{(v)}$ are defined for each value of a heavy baryon 4 -velocity $v^{\mu}$ as can be seen from Eq. (5.5). The redefinition in Eq. (5.5) has the effect of removing both the heavy quark mass, $m_{Q}$, and the nucleon mass, $m_{N}$, from the dynamics, leaving a dynamical scale of order $\lambda^{0}$, i.e., the scale of the interaction Hamiltonian near the combined limit which is $\mathcal{H}_{\text {int }}=\mathcal{H}-\left(m_{Q}+m_{N}\right)$.

The heavy quark part of the QCD Lagrangian density in terms of the fields $h_{Q}^{(v)}$ and $H_{Q}^{(v)}$ has the form,

$$
\begin{aligned}
\mathcal{L}_{Q} & =\bar{Q}\left(i \not D-m_{Q}\right) Q=\bar{h}_{Q}^{(v)}\left(i v^{\mu} D_{\mu}\right) h_{Q}^{(v)}-\bar{h}_{Q}^{(v)}\left(i v^{\mu} D_{\mu}+2 m_{Q}\right) H_{Q}^{(v)}+\bar{h}_{Q}^{(v)}\left(i D_{\perp}-2 m_{Q}\right) H_{Q}^{(v)} \\
& +\bar{H}_{Q}^{(v)}\left(\not D D_{\perp}\right) H_{Q}^{(v)}+m_{N}\left(\bar{h}_{Q}^{(v)} h_{Q}^{(v)}-\bar{H}_{Q}^{(v)} H_{Q}^{(v)}+\bar{H}_{Q}^{(v)} h_{Q}^{(v)}-\bar{h}_{Q}^{(v)} H_{Q}^{(v)}\right)
\end{aligned}
$$

where $D_{\perp}=D-\left(v^{\nu} D_{\nu}\right) v$ is the "transverse part" of the covariant derivative $D$. The heavy quark Lagrangian in Eq. (5.6) written in terms of the fields $h_{Q}^{(v)}$ and $H_{Q}^{(v)}$ differs from its analog in HQET due to the additional term proportional to $m_{N}$. This $m_{N}$ term would naively suggest that the fields $h_{Q}^{(v)}$ and $H_{Q}^{(v)}$ are both heavy with masses $m_{N}$ and $m_{Q}+m_{N}$, respectively, which apparently prevents integrating out the "heavy" field $H_{Q}^{(v)}$.

This problem is easily resolved if one considers the full Lagrangian density of the system:

$$
\mathcal{L}=\mathcal{L}_{Q}+\mathcal{L}_{q}+\mathcal{L}_{Y M}=\bar{Q}\left(i \not D-m_{Q}\right) Q+\sum_{j} \bar{q}_{j}\left(i \not D-m_{j}\right) q_{j}+\mathcal{L}_{Y M}
$$

where the sum is over all light quarks, and $\mathcal{L}_{Y M}$ is the Yang-Mills Lagrangian density. The total Lagrangian density in Eq. (5.7) should be re-expressed so as to build the brown muck contribution into the heavy degrees of freedom. This is completely analogous to the Hamiltonian treatment in Sec. III. In that case it is done by adding and subtracting to the Hamiltonian a quantity which is an overall constant $m_{N}$ and regrouping terms according to their $\lambda$ counting scaling. In the Lagrangian formalism this can be accomplished in the following way using a Lorentz covariant operator $m_{N} \bar{Q} \not \not Q:$ 


$$
\mathcal{L}=\left(\mathcal{L}_{Q}-m_{N} \bar{Q} \not \phi Q\right)+\left(\mathcal{L}_{q}+m_{N} \bar{Q} \not \phi Q+\mathcal{L}_{Y M}\right)=\mathcal{L}_{H}+\mathcal{L}_{\ell}
$$

where

$$
\mathcal{L}_{H} \equiv \mathcal{L}_{Q}-m_{N} \bar{Q} \not Q Q
$$

and

$$
\mathcal{L}_{\ell} \equiv \mathcal{L}_{q}+m_{N} \bar{Q} \not Q Q+\mathcal{L}_{Y M}
$$

In the rest frame of a heavy baryon operator, $\bar{Q} \not Q$ is equal to the heavy quark density operator $Q^{\dagger} Q$ and its spatial integral equals to one in the Hilbert space of heavy baryons. Thus, in the Hamiltonian the additional term corresponds to removing from the light degrees of freedom an overall constant $m_{N}$.

The additional operator proportional to the nucleon mass $m_{N}$ can easily be expressed in terms of the fields $h_{Q}^{(v)}$ and $H_{Q}^{(v)}$ :

$$
m_{N} \bar{Q} \not \phi Q=m_{N}\left(\bar{h}_{Q}^{(v)} h_{Q}^{(v)}-\bar{H}_{Q}^{(v)} H_{Q}^{(v)}+\bar{H}_{Q}^{(v)} h_{Q}^{(v)}-\bar{h}_{Q}^{(v)} H_{Q}^{(v)}\right) .
$$

This operator cancels the last term in Eq. (5.6) so that the Lagrangian density $\mathcal{L}_{H}$ has the form:

$$
\mathcal{L}_{H}=\bar{h}_{Q}^{(v)}\left(i v^{\mu} D^{\nu}\right) h_{Q}^{(v)}-\bar{h}_{Q}^{(v)}\left(i v^{\mu} D^{\nu}+2 m_{Q}\right) H_{Q}^{(v)}+\bar{h}_{Q}^{(v)}\left(\not D_{\perp}-2 m_{Q}\right) H_{Q}^{(v)}+\bar{H}_{Q}^{(v)}\left(\not D_{\perp}\right) H_{Q}^{(v)},
$$

where the condition $\not h_{Q}^{(v)}=h_{Q}^{(v)}$ is used. Thus, the "large" component $h_{Q}^{(v)}$ describes a massless field while the "small" component $H_{Q}^{(v)}$ has the mass $2 m_{Q}$. Using equations of motion the "heavy" field $H_{Q}^{(v)}$ can be expressed in terms of the "light" field $h_{Q}^{(v)}$ as follows:

$$
H_{Q}^{(v)}=\frac{1}{2 m_{Q}+i v^{\mu} D_{\mu}} i \not D_{\perp} h_{Q}^{(v)}
$$

The relation between fields $h_{Q}^{(v)}$ and $H_{Q}^{(v)}$ in Eq. (5.10) is identical in form to that in HQET between the "large" and "small" components of the heavy quark field $Q$.

We can now expand the field $Q=e^{i\left(m_{Q}+m_{N}\right) v^{\mu} y_{\mu}}\left(h_{Q}^{(v)}+H_{Q}^{(v)}\right)$, the Lagrangian density in Eq. (5.9), and the current $J=\bar{Q}_{j} \Gamma Q_{i}$ in powers of $\lambda$. This can be done by eliminating fields $H_{Q}^{(v)}$ (using Eq. (5.10)) and expanding the numerator in powers of $\left(i v^{\mu} D_{\mu}\right) / 2 m_{Q}$. Thus, the combined expansion of the heavy quark field including terms up to order $\lambda$ is,

$$
Q(y)=e^{-i\left(m_{Q}+m_{N}\right) v^{\mu} y_{\mu}}\left[1+\left(\frac{1-\not p}{2}\right) \frac{i \not D}{2 m_{Q}}\right] h_{Q}^{(v)}(y)+\mathcal{O}\left(\lambda^{2}\right) .
$$

Using this expansion the Lagrangian density $\mathcal{L}_{H}$ including terms up to order $\lambda$ has the form:

$$
\mathcal{L}_{H}=\bar{h}_{Q}^{(v)}\left(i v^{\mu} D_{\mu}\right) h_{Q}^{(v)}+\frac{1}{2 m_{Q}} \bar{h}_{Q}^{(v)}\left[-\left(i v^{\mu} D_{\mu}\right)^{2}+(i D)^{2}-\frac{1}{2} g_{s} \sigma_{\mu \nu} G^{\mu \nu}\right] h_{Q}^{(v)}+\mathcal{O}\left(\lambda^{2}\right),
$$

where $g_{s}$ is a strong coupling constant and $G^{\mu \nu}=\left[D^{\mu}, D^{\nu}\right]$ is the gluon field strength tensor. In a similar way we can expand the current $J=\bar{Q}_{j} \Gamma Q_{i}$; again keeping terms up to $\lambda$ we get,

$$
J(y=0)=\bar{h}_{Q_{j}}^{(v)} \Gamma h_{Q_{i}}^{(v)}+\frac{1}{2 m_{Q_{i}}} \bar{h}_{Q_{j}}^{(v)} \Gamma(i \not D) h_{Q_{i}}^{(v)}+\frac{1}{2 m_{Q_{j}}} \bar{h}_{Q_{i}}^{(v)}(-i \overleftarrow{\not D}) \Gamma h_{Q_{i}}^{(v)}+\mathcal{O}\left(\lambda^{2}\right) .
$$

The expressions in Eqs. (5.11), (5.12), (5.13) are identical in form to the analogous quantities in HQET. However, there is an important difference: these expressions represent the combined expansion in powers of $\lambda$ and not the $1 / m_{Q}$ expansion of HQET. In other words, by defining fields $h_{Q}^{(v)}$ and $H_{Q}^{(v)}$ appropriately we were able to resum implicitly all the $m_{N} / m_{Q}$ corrections in HQET.

The correction terms in the combined expansion of the electroweak current, Eq. (5.13), come at order $\lambda$ and not at $\lambda^{1 / 2}$. It will be shown in Ref. [3] that these corrections contribute to the matrix elements at order $\lambda$ as well, i.e., they do not induce any anomalously large constants which violate the combined power counting of these operators. Thus, 
at NLO in our expansion only the leading order operator, $\bar{h}_{Q_{j}}^{(v)} \Gamma h_{Q_{i}}^{(v)}$, contributes to the matrix elements. In addition, it will be shown in Ref. [3] that in the combined limit at NLO there is only a single form factor which parameterizes the matrix elements of the current $J$ in the $\Lambda_{Q}$ sector. Accordingly, we can consider one Lorentz component of the current $J$, namely the $\mu=0$ component. Thus, to completely determine the matrix elements at NLO in the combined expansion, we need to know the form of only one operator, namely $h_{Q_{j}}^{(v) \dagger} h_{Q_{i}}^{(v)}$.

Let us first consider the flavor conserving operator $h_{Q}^{(v) \dagger}(\vec{y}) h_{Q}^{(v)}(\vec{y})$. As we are working near the heavy quark limit it is legitimate to expand the state in a fock-space decomposition for the number of heavy quarks and antiquarks. For a heavy baryon state standard heavy quark analysis implies that the leading fock component has one heavy quark; the first sub-leading fock component with two quarks and one antiquark is suppressed by a factor of $\Lambda / m_{Q} \sim \lambda$. Thus at NLO in $\lambda^{1 / 2}$ we can neglect the sub-leading fock component and treat the state as having a single heavy quark. This implies that acting in our heavy baryon Hilbert space, the operator $h_{Q}^{(v) \dagger}(\vec{y}) h_{Q}^{(v)}(\vec{y})$ is $\delta^{3}\left(\vec{X}_{Q}-\vec{y}\right)$. Since this is true at the QCD level at NLO it immediately holds in the effective theory at that order.

In order to describe the flavor changing current we need to enlarge the Hilbert space of our effective theory to account for electroweak transitions between heavy baryons with heavy quarks of different flavors. The new Hilbert space is the direct product of the Hilbert spaces for baryons with a given flavor of a heavy quark described in Sec. III. Any state of the enlarged Hilbert space can be written as $n_{f}$-dimensional vector, where $n_{f}$ is a number of heavy flavors. Each component of this vector describes a state with a given flavor. The effective Hamiltonian can be readily generalized to include baryons of various heavy quark flavors. It can be written as $n_{f} \times n_{f}$ diagonal matrix. For example, for two heavy flavors (e.g. charm and bottom) the effective Hamiltonian including terms up to NLO in $\lambda^{1 / 2}$ has the form:

$$
H_{\mathrm{eff}}=\left(\begin{array}{cc}
\mathcal{H}_{Q_{j}} & 0 \\
0 & \mathcal{H}_{Q_{i}}
\end{array}\right)+\mathcal{O}\left(\lambda^{3 / 2}\right)
$$

where the diagonal entries $\mathcal{H}_{Q_{j}}$ and $\mathcal{H}_{Q_{i}}$ are the effective Hamiltonians as in Eq. 4.5). Analogously, QCD operators acting in the enlarged Hilbert space become $n_{f} \times n_{f}$ matrices. The flavor changing weak transitions can be described by off-diagonal matrix elements of an operator $h_{Q_{j}}^{(v) \dagger}(\vec{y}) h_{Q_{i}}^{(v)}(\vec{y})$, where the subscripts $i$ and $j$ can take integer values from 1 to $n_{f}$. This operator can be written in terms of collective operators only. It follows from arguments analogous to the case of the flavor conserving electroweak operator $-h_{Q}^{(v) \dagger}(\vec{y}) h_{Q}^{(v)}(\vec{y})$ - that the flavor changing operator acting on the enlarged Hilbert space has the form (for $n_{f}=2$ ):

$$
h_{Q_{j}}^{(v) \dagger}(\vec{y}) h_{Q_{i}}^{(v)}(\vec{y})=\left(\begin{array}{ll}
\delta^{3}\left(\vec{X}_{Q_{j}}-\vec{y}\right) & \delta^{3}\left(\vec{X}_{Q_{j}}-\vec{y}\right) \\
\delta^{3}\left(\vec{X}_{Q_{i}}-\vec{y}\right) & \delta^{3}\left(\vec{X}_{Q_{i}}-\vec{y}\right)
\end{array}\right)+\mathcal{O}(\lambda) .
$$

The diagonal elements of the operator in Eq. (5.15) correspond to flavor conserving operators, while the off-diagonal elements correspond to flavor changing operators. Their action is to instantaneously change the heavy quark flavor at the position where a heavy baryon interacts with the current. Since the operator in Eq. (5.15) is written in terms of the collective operators only, the corresponding effective operator has an identical form.

Finally, let us consider the operator defined by

$$
\vec{d}_{\ell}=\frac{1}{N_{c}-1} \sum_{i=\text { light }} \int d^{3} x q_{i}^{\dagger}(\vec{x}) \vec{x} q_{i}(\vec{x})
$$

so that $\vec{d}_{\ell}$ represents the contribution to the baryon dipole moment coming from light quarks. In calculating electromagentic transitions between heavy baryons it is useful to divide the electromagnetic current into pieces coming from the heavy and the light quarks and to further divide the light quark contribution into isovector and isoscalar pieces. The isoscalar piece is simply the light-quark contribution to the baryon current. Thus, matrix elements of $\vec{d}_{\ell}$ must be calculated to describe electric dipole transitions of heavy baryons.

At first sight, it might appear to be impossible to express the operator $\vec{d}_{\ell}$ in terms of collective operators. Although $\vec{d}_{\ell}$ - the collective position operator for light quarks - is intuitively something like the position of the brown muck and clearly transforms in the same way as $\vec{X}_{\ell}$, at the operator level $\vec{d}_{\ell}$ is manifestly not identical to $\vec{X}_{\ell}$. Recall that by construction $m_{N} \vec{X}_{\ell}$ acts as a generator of boosts for the light degrees of freedom (up to order $\lambda$ corrections). On the other hand, the operator $\vec{d}_{\ell}$ acts only on the quarks and not on the gluons. Thus $m_{N} \vec{d}_{\ell}$ cannot boost the gluons and therefore does not act as a boost for the light degrees of freedom (which include both quarks and gluons). Thus, the operator $\vec{d}_{\ell}$ is clearly distinct from $\vec{X}_{\ell}$ and there is apparently no simple way to express $\vec{d}_{\ell}$ entirely in terms of collective operators. 
Remarkably, however, one can show that

$$
\left\langle\psi^{\prime}\left|\vec{d}_{\ell}\right| \psi\right\rangle=\left\langle\psi^{\prime}\left|\vec{X}_{\ell}\right| \psi\right\rangle
$$

for all $|\psi\rangle,\left|\psi^{\prime}\right\rangle$ in the collective subspace defined in Sec. III. Thus, when one restricts consideration to the collective subspace which dominates the low energy physics, $\vec{d}_{\ell}$ is equivalent to $\vec{X}_{\ell}$.

To see how this comes about, we start by considering the commutation relations of $\vec{d}_{\ell}$ with $\vec{P}_{\ell}, \vec{X}_{Q}$ and $\vec{P}_{Q}$. To determine the commutator with $\vec{P}_{\ell}$ we exploit the fact that $\vec{P}_{\ell}$ is a generator of translations for the light degrees of freedom so that $\exp \left(i \vec{P}_{\ell} \cdot \vec{\Delta}\right) q(\vec{x}) \exp \left(-i \vec{P}_{\ell} \cdot \vec{\Delta}\right)=q(\vec{x}-\vec{\Delta})$, where $q$ is a generic light quark field. We can use this to deduce that

$$
\begin{aligned}
& \exp \left(i \vec{P}_{\ell} \cdot \vec{\Delta}\right)\left(\vec{d}_{\ell}\right) \exp \left(-i \vec{P}_{\ell} \cdot \vec{\Delta}\right)=\exp \left(i \vec{P}_{\ell} \cdot \vec{\Delta}\right)\left(\frac{1}{N_{c}-1} \sum_{i=\text { light }} \int d^{3} x q_{i}^{\dagger}(\vec{x}) \vec{x} q_{i}(\vec{x})\right) \exp \left(-i \vec{P}_{\ell} \cdot \vec{\Delta}\right) \\
= & \left(\frac{1}{N_{c}-1} \sum_{i=\text { light }} \int d^{3} x q_{i}^{\dagger}(\vec{x}-\vec{\Delta}) \vec{x} q_{i}(\vec{x}-\vec{\Delta})\right)=\left(\frac{1}{N_{c}-1} \sum_{i=\text { light }} \int d^{3} x^{\prime} q_{i}^{\dagger}\left(\vec{x}^{\prime}\right)\left(\vec{x}^{\prime}+\vec{\Delta}\right) q_{i}\left(\vec{x}^{\prime}\right)\right) \\
= & \left(\frac{1}{N_{c}-1} \sum_{i=\text { light }} \int d^{3} x^{\prime} q_{i}^{\dagger}\left(\vec{x}^{\prime}\right) \vec{x}^{\prime} q_{i}\left(\vec{x}^{\prime}\right)+\vec{\Delta} \frac{1}{N_{c}-1} \sum_{i=\text { light }} \int d^{3} x^{\prime} q_{i}^{\dagger}\left(\vec{x}^{\prime}\right) q_{i}\left(\vec{x}^{\prime}\right)\right)=\vec{d}_{\ell}+\vec{\Delta} .
\end{aligned}
$$

The first equality in Eq. (5.18) follows from the definition of $\vec{d}_{\ell}$, the second equality follows because $\vec{P}_{\ell}$ translates the light quark degrees of freedom, the third equality follows from a change of variables, and the final equality follows from the definition of $\vec{d}$ and the fact that

$$
\sum_{i=\text { light }} \int d^{3} x^{\prime} q_{i}^{\dagger}\left(\vec{x}^{\prime}\right) q_{i}\left(\vec{x}^{\prime}\right)
$$

counts the net number of light quarks, which is $N_{c}-1$ in a heavy baryon containing one heavy quark. Equation (5.18) implies that

$$
\left[d_{\ell j}, P_{\ell k}\right]=i \delta_{j k}
$$

while the fact that from its definition $\vec{d}_{\ell}$ has no heavy quark operators implies that

$$
\left[d_{\ell j}, X_{Q_{j}}\right]=0,\left[d_{\ell j}, P_{Q_{k}}\right]=0 .
$$

Thus, the commutation relations of $\vec{d}_{\ell}$ with $\vec{P}_{\ell}, \vec{X}_{Q}$ and $\vec{P}_{Q}$ are identical to the commutation relations of $\vec{X}_{\ell}$ with $\vec{P}_{\ell}$, $\vec{X}_{Q}$ and $\vec{P}_{Q}$. In Eqs. (5.19), (5.20) indices $i, j=1,2,3$ indicate Cartesian components of $\vec{X}_{\ell}, \vec{P}_{\ell}, \vec{X}_{Q}$ and $\vec{P}_{Q}$.

Next let us express $\overrightarrow{d_{\ell}}$ in terms of the various collective and non-collective operators in the problem. Generically, one can represent an arbitrary operator $\mathcal{O}$ as a power series in our collective operators:

$$
\mathcal{O}=\sum_{N} \hat{b}_{N}^{\mathcal{O}} \prod_{i=1,2,3} P_{\ell_{i}}^{N_{P_{\ell_{i}}}} \prod_{i=1,2,3} X_{\ell_{i}}^{N_{X_{\ell_{i}}}} \prod_{i=1,2,3} P_{Q_{i}}^{{ }^{N_{P_{Q_{i}}}}} \prod_{i=1,2,3} X_{Q_{i}}^{{ }^{N_{X_{Q_{i}}}}}
$$

with $N \equiv\left(N_{P_{\ell 1}}, N_{P_{\ell 2}}, N_{P_{\ell 3}}, N_{X_{\ell 1}}, N_{X_{\ell 2}}, N_{X_{\ell 3}}, N_{P_{Q_{1}}}, N_{P_{Q_{2}}}, N_{P_{Q_{3}}}, N_{X_{Q_{1}}}, N_{X_{Q_{2}}}, N_{X_{Q_{3}}}\right)$ specifying a particular term in the expansion, and $i=1,2,3$ indicating the three Cartesian directions. The coefficients in the expansion, $\hat{b}_{N}^{\mathcal{O}}$, are generally non-collective operators which commute with $\vec{X}_{\ell}, \vec{P}_{l}, \vec{X}_{Q}$ and $\vec{P}_{Q}$. Expanding one component of $\vec{d}_{\ell}$ in this form gives

$$
d_{\ell j}=X_{\ell j}+\sum_{N_{P_{\ell}}, N_{P_{\ell 2}}, N_{P_{\ell 3}}} \hat{b}_{N_{P_{\ell 1}}, N_{P_{\ell 2}}, N_{P_{\ell 3}}}^{d_{\ell_{j}}} \prod_{i=1,2,3} P_{\ell_{i}}^{N_{P_{\ell i}}},
$$

where the fact that the $\vec{d}_{\ell}$ commutes with $\vec{X}_{Q}$ or $\vec{P}_{Q}$ impose the restriction that no nonzero powers of $\vec{X}_{Q}$ or $\vec{P}_{Q}$ can contribute to the series, while the commutation relation of Eq. (5.19) implies that the only contributing term containing any powers of $\vec{X}_{\ell}$ is the linear term and that the coefficient of this term is unity. 
The known parity and time reversal properties of $\vec{d}_{\ell}$ and $\vec{P}_{\ell}$ fix the parity and time reversal properties of the non-collective coefficient operators, $\hat{b}_{N_{P_{\ell_{1}}}, N_{P_{\ell_{2}}}, N_{P_{\ell_{3}}}}$. Since $\vec{d}_{\ell}$ is time reversal even and parity odd while $\vec{P}_{\ell}$ is time reversal odd and parity even, it follows that:

$$
\begin{aligned}
& T\left(\hat{b}_{N_{P_{\ell 1}}} d_{\ell_{P_{\ell 2}}}, N_{P_{\ell 3}}\right)=(-1)^{N_{P_{\ell 1}}+N_{P_{\ell 2}}+N_{P_{\ell 3}}} \\
& P\left(\hat{b}_{N_{P_{\ell 1}}} d_{\ell_{P_{\ell 2}}}, N_{P_{\ell 3}}\right)=(-1)^{N_{P_{\ell 1}}+N_{P_{\ell 2}}+N_{P_{\ell 3}}+1}
\end{aligned}
$$

The significant point about Eq. (5.23) is that every non-collective coefficient operator is odd under PT. From the analysis of Sec. III, generic states can be written as superpositions of outer product states containing collective and intrinsic parts. The collective subspace of the theory is the subspace where the intrinsic wave function is in its ground state. Thus, an arbitrary state in the collective subspace can be written as $|\psi\rangle=\left|\psi_{C}\right\rangle \otimes\left|0_{I}\right\rangle$ where $0_{I}$ indicates the ground state of the intrinsic system. Now consider the matrix element of a typical term in the series of Eq. (5.22) between two collective states:

$$
\left\langle\psi^{\prime}\left|\hat{b}_{N_{P_{\ell 1}}, N_{P_{\ell 2}}, N_{P_{\ell 3}}} \prod_{i=1,2,3} P_{\ell_{i}}^{N_{P_{\ell_{i}}}}\right| \psi\right\rangle=\left\langle\psi_{C}^{\prime}\left|\prod_{i=1,2,3} P_{\ell_{i}}^{N_{P_{\ell_{i}}}}\right| \psi_{C}\right\rangle\left\langle 0_{I}\left|\hat{b}_{N_{P_{\ell_{1}}}, N_{P_{\ell_{2}}}, N_{P_{\ell 3}}}^{d_{I}}\right| 0_{I}\right\rangle
$$

where we have separated the matrix element into its collective and intrinsic parts. Clearly, the matrix element in Eq. (5.24) vanishes: the intrinsic part of the of the matrix element is a diagonal matrix element of a PT odd operator in a state of good PT. Thus, within the collective subspace all terms in the series of Eq. (5.22) vanish except the first term. This implies that within this subspace all matrix elements of $\vec{d}_{\ell}$ are identical to matrix elements of $\vec{X}_{\ell}$ and Eq. (5.17) is established.

As written, the equivalence expressed in Eq. (5.17) only holds for states in the collective subspace, i.e., states with the intrinsic subspace in its ground state. As discussed in Sec. IV, the non-collective operators in the Hamiltonian induce components with excited intrinsic states in the physical low-energy states. This in turn spoils the formal equivalence in Eq. (5.17) for the physical states. Fortunately, however, it is straightforward to see that in the physical states, the matrix elements of $\vec{d}_{\ell}$ differ from those of $\vec{X}_{\ell}$ by an amount of order $\lambda$ (or less) and hence may be neglected at next-to-leading order in $\lambda^{1 / 2}$. In the first place using reasoning analogous to that leading to Eq. (3.5), it is easy to see that the matrix elements of the $\hat{b}_{N_{P_{\ell}}, N_{P_{\ell 2}}, N_{P_{\ell 3}}}^{d_{\ell_{j}}}$ between the intrinsic ground state and its excited states is of order $\lambda^{1 / 2}$. On the other hand, the analysis of Sec. IV implies that admixtures of excited intrinsic states in the physical states are suppressed by at least order $\lambda^{1 / 2}$. Combining these two facts one sees that the matrix elements of $\vec{d}_{\ell}$ and $\vec{X}_{\ell}$ in the physical states are equivalent at NLO.

The equivalence at NLO between the matrix elements of $\vec{d}_{\ell}$ and $\vec{X}_{\ell}$ may seem paradoxical. After all, as discussed above, the two operators are clearly quite distinct at the QCD level. It would seem that the fact that the operators are different would imply that the matrix elements of the two operators should be different in general. The resolution of the paradox is quite simple: the matrix elements of the two operators are different in general. They only coincide (up to NNLO corrections) for a very special class of states - the collective states with excitation energies of order $\lambda^{1 / 2}$. For states with excitation energies of order unity, the matrix elements of the two operators can be quite different. Fortunately, we will be using our effective theory to study these low-lying excitations only. Thus, for the purpose of the effective theory at NLO, it is legitimate to replace $\vec{d}_{\ell}$ with $\vec{X}_{\ell}$.

\section{SUMMARY}

In this paper we have derived an effective theory which will be used in Ref. 3] to study the spectroscopy and electroweak decays of the low-energy states of heavy baryons. The analysis is based largely on the formulation of the problem in Ref. [2]. A power-counting scheme is developed in $\lambda^{1 / 2}$, where $\lambda \sim 1 / N_{c}, \Lambda / m_{Q}$. The kinematical variables of the problem $\vec{X}, \vec{P}, \vec{x}$ and $\vec{p}$ are directly related to QCD operators which are well defined up to corrections at relative order $\lambda$. This formalism is based on standard large $N_{c}$ and heavy quark analysis.

At the theoretical level, the analysis used in our derivation of the effective theory goes well beyond the analysis of Ref. [2] which was largely kinematical and group-theoretical in nature. In our derivation, the structure of the Hilbert space for heavy baryons plays a critical role. In particular, as the $\lambda \rightarrow 0$ limit is approached, the heavy baryon states can be written as products of collective states (with excitation energies of order $\lambda^{1 / 2}$ ) and intrinsic states (with excitation energies of order $\lambda^{0}$ ). To deduce this form of the Hilbert space we exploited the fact that our formulation 
as an expansion in $\lambda$ does not depend formally on whether the large $N_{c}$ limit is taken first, the heavy quark limit is taken first or they are taken simultaneously. By taking the large $N_{c}$ limit first we can use standard large $N_{c}$ reasoning to deduce the structure of the Hilbert space and then map back to the case where the limits are taken concurrently. One essential point in this analysis is that matrix elements of operators which couple between different intrinsic states are characteristically down by a factor of $\lambda^{1 / 2}$ compared to matrix elements of operators with the same intrinsic state. Thus, the effect of coupling to an excited intrinsic state and then back to the ground state is typically of order $\lambda$ and hence can be neglected at NLO in the $\lambda^{1 / 2}$ expansion.

From the kinematics of the problem, the underlying heavy quark theory, Poincare invariance, and the structure of the Hilbert space, we showed that the effective Hamiltonian up to order $\lambda$ can be written in the form of Eq. (4.5). At order $\lambda^{1 / 2}$ one sees that the excitation spectrum is fixed entirely by a single parameter $\kappa$, and at order $\lambda$ only one additional parameter - $\alpha$ - enters. This is encouraging from a phenomenological perspective since we do not have a large number of coefficients to work with at NLO.

We also derived the certain effective operators at NLO. It turns out that the operators discussed here are precisely those needed to compute interesting electroweak transitions of isoscalar heavy baryons at NLO. The key point in the derivation is that all of these operators can be written in terms of the collective operators (up to possible NNLO corrections). From this it was easy to show that the effect of the admixture of components only comes in at NNLO so that at NLO one can simply replace the QCD level collective operator by an effective operator of the same structure. One operator, $\vec{d}_{\ell}$, which is effectively the dipole moment of the light quark contribution to the baryon density, was somewhat more subtle. Although, as an operator it cannot be written directly in terms of the collective variables, we showed that its matrix elements between low-lying states are identical to those of the collective operator $\vec{X}_{\ell}$.

The effective theory derived here is suitable for the study of isoscalar heavy baryons at NLO in our $\lambda^{1 / 2}$ expansion. In Ref. [3] we will use the effective Hamiltonian of Sec. IV to study the spectroscopy and electroweak decays of $\Lambda_{c}$ and $\Lambda_{b}$ baryons and their excited states. In order to calculate the semi-leptonic matrix elements we will use relations between form-factors analogous to those obtained in HQET 20 25]. These matrix elements can then be determined using the effective operators discussed in Sec. $\mathrm{V}$. We will also calculate the radiative decay rates of the first excited states of $\Lambda_{Q}$ baryons.

\section{ACKNOWLEDGMENTS}

We wish to acknowledge the support of the U.S. Department of Energy under Grant No. DE-FG02-93ER-40762.

[1] C.K. Chow and T.D. Cohen, Phys. Rev. Lett. 845474 (2000).

[2] C.K. Chow and T.D. Cohen, hep-ph/0003131, Nucl. Phys. A to be published.

[3] A. Baccouche, C.K. Chow, T.D. Cohen and B.A. Gelman, "Excited Heavy Baryons and Their Symmetries III: Phenomenology", DOE/ER/40762-215, UM PP\#01-029, in preparation.

[4] Z. Guralnik, M. Luke and A.V. Manohar, Nucl. Phys. B390 474 (1993).

[5] E. Jenkins, A.V. Manohar and M.B. Wise, Nucl. Phys. B396 27 (1993);

[6] E. Jenkins, A.V. Manohar and M.B. Wise, Nucl. Phys. B396 38 (1993).

[7] C.K. Chow and M.B. Wise, Phys. Rev. D50 2135 (1994).

[8] M. Rho, D.O. Riska and N.N. Scoccola, Z. Phys. A341 343 (1992);

[9] D.P. Min, Y. Oh, B.Y. Park and M. Rho, Int. J. Mod. Phys. E4 47 (1995);

[10] J. Schechter, A. Subbaraman, S. Vaidya and H. Weigel, Nucl. Phys. A590 655 (1995);

[11] L.Ya. Glozman and D.O. Riska, Nucl. Phys. A603 326 (1996);

[12] M. Harada, F. Sannino, J. Schechter and H. Weigel, Phys. Rev. D56 4098 (1997).

[13] J.L. Gervais and B. Sakita, Phys. Rev. Lett. 5287 (1984).

[14] J.L. Gervais and B. Sakita, Phys. Rev. D30 1795 (1984).

[15] R. Dashen, E. Jenkins and A.V. Manohar, Phys. Rev. D49 4713 (1994).

[16] C. Carone, H. Georgi, S. Osofsky, Phys. Lett. B322 227 (1994).

[17] M. Luty and J. March-Russell, Nucl. Phys. B426 71 (1994).

[18] G. 't Hooft, Nucl. Phys. B72 461 (1974).

[19] E. Witten, Nucl. Phys. B160 57 (1979). 
[20] N. Isgur and M.B. Wise, Phys. Lett. B232 113 (1989).

[21] N. Isgur and M.B. Wise, Phys. Lett. B237 527 (1990).

[22] N. Isgur and M.B. Wise, Nucl. Phys. B348 276 (1991).

[23] H. Georgi, Nucl. Phys. B348 293 (1991).

[24] T. Mannel, W. Roberts and Z. Ryzak, Nucl. Phys. B355 38 (1991).

[25] F. Hussain, J.G. Korner, M. Kramer and G. Thompson, Z. Phys. C51 321 (1991).

[26] M.E. Luke, Phys. Lett. B252 447 (1990).

[27] H. Georgi, B. Grinstein and M.B. Wise, Phys. Lett. B252 456 (1990).

[28] A.F. Falk, B. Grinstein and M.E. Luke, Nucl. Phys. B357 185 (1991).

[29] M. Neubert, Phys. Rev. D49 1542 (1994). 